LAWRENCE LIVERMORE N A T IO N A L LABORATORY

\section{An Implicit Algorithm for the Numerical Simulation of Shape-Memory Alloys}

C. Jannetti, R. Becker, J. Stolken, J. Bassani

February 13, 2004 
This document was prepared as an account of work sponsored by an agency of the United States Government. Neither the United States Government nor the University of California nor any of their employees, makes any warranty, express or implied, or assumes any legal liability or responsibility for the accuracy, completeness, or usefulness of any information, apparatus, product, or process disclosed, or represents that its use would not infringe privately owned rights. Reference herein to any specific commercial product, process, or service by trade name, trademark, manufacturer, or otherwise, does not necessarily constitute or imply its endorsement, recommendation, or favoring by the United States Government or the University of California. The views and opinions of authors expressed herein do not necessarily state or reflect those of the United States Government or the University of California, and shall not be used for advertising or product endorsement purposes.

This work was performed under the auspices of the U.S. Department of Energy by University of California, Lawrence Livermore National Laboratory under Contract W-7405-Eng-48. 


\title{
An Implicit Algorithm for the Numerical Simulation of Shape-Memory Alloys
}

\author{
Carl Jannetti ${ }^{1}$, Rich Becker ${ }^{2}$, James Stölken ${ }^{2}$, and John Bassani ${ }^{1}$ \\ ${ }^{1}$ Department of Mechanical Engineering and Applied Mechanics, \\ University of Pennsylvania \\ ${ }^{2}$ New Technologies Engineering Division, \\ Lawrence Livermore National Laboratory
}

August 12, 2003

\begin{abstract}
Shap- memory alloys (SMA) have the potential to be used in a variety of interesting applications due to their unique properties of pseudoelasticity and the shape-memory effect. However, in order to design SMA devices efficiently, a physics-based constitutive model is required to accurately simulate the behavior of shape-memory alloys. The scope of this work is to extend the numerical capabilities of the SMA constitutive model developed by Jannetti et. al. (2003), to handle large-scale polycrystalline simulations. The constitutive model is implemented within the finite-element software ABAQUS/ Standard using a user defined material subroutine, or UMAT. To improve the efficiency of the numerical simulations, so that polycrystalline specimens of shape-memory alloys can be modeled, a fully implicit algorithm has been implemented to integrate the constitutive equations. Using an implicit integration scheme increases the efficiency of the UMAT over the previously implemented explicit integration method by a factor of more than 100 for single crystal simulations. This work was performed under the auspices of the U.S. Department of Energy by the University of California, Lawrence Livermore National Laboratory under contract No. W-7405-Eng-48.
\end{abstract}

\section{Introduction}

Shape-memory alloys (SMA) exhibit unique macroscopic properties that result from a reversible solid-to-solid diffusionless phase transformation. The macroscopic properties that result from this microstructural rearrangement are pseudoelasticity and the shape-memory effect. These properties make shape-memory alloys excellent candidates for a variety of applications. At the present time, SMAs are used in many applications such as eyeglass frames, arterial stents, as well as sensors and actuators.

The scope of the work presented here is to use the three-dimensional, inelastic, continuum-level constitutive description of the phase transformation that occurs in shapememory alloys developed by Jannetti et. al. (2003) to simulate polycrystal SMA specimens. To perform numerical simulations, the constitutive model had been previously implemented in ABAQUS/Standard (2003) using a user defined material behavior subroutine (UMAT). 
However, the previous implementation of the UMAT was not sufficient to perform large-scale polycrystal simulations due to the small time steps required as a result of the explicit integration scheme employed to integrate the constitutive equations. The work described here is the implementation of an implicit time integration scheme that allows for much larger time steps to be taken and larger scale simulations to be performed. The ability to perform large-scale polycrystal simulations of SMA specimens is important so that SMA devices can be efficiently and optimally designed.

The phase transformation in shape-memory alloys, which is described by the constitutive model and simulated by the UMAT, is similar to the transformation that occurs when high temperature steel is quenched. The fine-scale rearrangement that occurs involves changing from an austenite crystal structure to a finer microstructure, referred to as martensite. The pseudoelastic and shape-memory effect exhibited macroscopically by the SMAs is a result of this solid-to-solid phase transformation that occurs at the microscale level.

Pseudoelastcity refers to the ability of the shape-memory alloy to be capable of full strain and structure recovery due to a complete reversal of the phase transformation from martensite to austenite. Nevertheless, this typically involves energy dissipation, i.e. hysteresis. Pseudoelasticity occurs when the SMA specimen is subjected to a loading-unloading cycle, without inducing any plastic deformation, while the temperature of the specimen is above the austenite finish temperature. Typical shape-memory alloy specimens may be subjected to uniaxial strains on the order of seven to ten percent and still exhibit full strain and structure recovery upon unloading. While the pseudoelastic process recovers the deformation, the cycle involves dissipation, as evidenced by the hysteresis loop in the stress-strain curve. The dissipation is related to the movement of interfaces between various phases of the material.

The shape-memory effect is due to the reverse transformation, which is due in part to the reversible nature of the self-accommodation by twining rather than slip, which occurs when the specimen is heated. When the a SMA specimen is deformed at a temperature below the austenite start temperature and above the martensite finish temperature, upon unloading the specimen will appear to be permanently deformed. However, if the specimen is subjected to a thermal cycle that includes heating the material to a temperature above the austenite finish temperature, the specimen recovers its original shape. 
The remainder of this document is outlined as follows: Section 2 briefly outlines the constitutive model used to describe the behavior of the shape-memory alloys, Section 3 describes the new implicit time integration scheme that is used to adapt the UMAT for large-scale simulations, Section 4 lists the future work to de done, Appendix I contains the UMAT source code, and Appendix II contains a sample ABAQUS/ Standard input file.

\section{Constitutive Model}

The constitutive behavior of SMAs that is used in the simulations is the model developed by Jannetti et. al. (2003). This model is a thermodynamically-based, three-dimensional, finite strain, continuum-level description of the material behavior of the SMAs. This model was developed following Rice's $(1971,1975)$ notion that the rate of any microscale rearrangement is taken to be dependent on the thermodynamic force conjugate to the rearrangement. The following subsections describe the major points of the constitutive model.

\subsection{Finite Strain Kinematics}

To describe the finite deformation, let $\mathbf{X}$ be the position vector of some material point in the reference configuration and let $\mathbf{x}=\mathbf{x}(\mathbf{X}, t)$ denote the position vector of that point in the current or deformed configuration. Let $\mathbf{F}$ be the be the deformation gradient such that

$$
\mathrm{d} \mathbf{x}=\mathbf{F d} \mathbf{X} .
$$

Since the deformation of shape-memory alloys is due to both elastic deformation and deformation due to the phase transformation, a multiplicative decomposition of the deformation gradient is used

$$
\mathbf{F}=\mathbf{F}^{\mathrm{e}} \mathbf{F}^{\mathrm{tr}}
$$

where $\mathbf{F}^{\mathrm{e}}$ is the deformation associated with the elastic distortion of the lattice and $\mathbf{F}^{\mathrm{tr}}$ is the deformation associated with the phase change.

\subsection{RVE}

To motivate the remaining sections on the kinematics of the phase transformation, a description of the material microstructure is necessary. Because the constitutive model is continuum level in nature, material points are assumed to have statistically homogeneous properties. On the other hand, at microscopic scales the underlying material will have spatially 
varying microstructure. To resolve this issue the notion of a representative volume element is used to characterize the microstructure, where the RVE is statically representative of the material neighborhood of that point, i.e. statistically homogeneous. In this constitutive model, the RVE is a region that may contain several phases of martensite embedded in austenite. The RVE can be thought of as a multi-phase composite with specific volume fractions that evolve with deformation. The volume fractions of the phases are denoted by $\xi^{\alpha}$, where $\alpha=0$ denotes the austenite phase and $\alpha=1, \ldots, N$ denotes the transformation systems of martensite, where

$$
\xi^{\alpha=0}=1-\sum_{\alpha=1}^{N} \xi^{\alpha} \equiv 1-\xi,
$$

with $\xi$ denoting the total volume fraction of the RVE (the vector of $\xi^{\alpha}$ 's is denoted by $\xi$ ). The following constraints apply to (3)

$$
0 \leq \xi \leq 1 \text { and } 0 \leq \xi^{\alpha} \leq 1 \text { for } \alpha=0, \ldots, N .
$$

Given the properties of the individual phases, the effective elastic response of such an RVE clearly depends on the volume fractions, $\xi^{\alpha}$, when the corresponding elastic properties of each phase are different. The homogenized elastic properties of the RVE is determined using a Reuss estimate (Reuss, 1929) as

$$
\tilde{\mathbf{C}}^{\mathrm{eff}}=\left((1-\xi) \tilde{\mathbf{M}}^{(\mathrm{a})}+\sum_{\alpha=1}^{N} \xi^{\alpha} \tilde{\mathbf{M}}^{(\mathrm{m})^{\alpha}}\right)^{-1},
$$

where $\tilde{\mathbf{C}}^{\text {eff }}(\xi)$ is the effective tensor of elastic moduli, $\tilde{\mathbf{M}}^{(\mathrm{a})}$ denotes the elastic compliance of the

austenite, and $\tilde{\mathbf{M}}^{(\mathrm{m})^{\alpha}}$ denotes the elastic compliance of each of the martensite transformation systems.

\subsection{Phase Transformation Kinematics}

If one considers the RVE as a multi-phase composite composed of austenite and several phases of martensite, each phase contributes to the overall deformation. Using classic averaging results for multi-phase composites, the volume average of the transformation deformation gradient, neglecting elastic deformation, can be written as

$$
\mathbf{F}^{\mathrm{tr}}=\mathrm{I}+\sum_{\alpha=1}^{N} \xi^{\alpha} \gamma_{0}^{\alpha}
$$


where $\gamma_{0}^{\alpha}$ is the second order tensor corresponding to the deformation associated with the transformation system $\alpha$, and $\mathrm{I}$ is the second order identity tensor. The $\gamma_{0}^{\alpha}$, s are expressed in terms of the unit normal to the habit plane (austenite-martensite interface), denoted by $\mathbf{m}_{0}^{\alpha}$, and the unit average transformation direction, denoted by $\mathbf{b}_{0}^{\alpha}$, and the magnitude of the transformation strain, $\gamma_{T}$. For the case of NiTi,

$$
\gamma_{0}^{\alpha}=\gamma_{T} \mathbf{b}_{0}^{\alpha} \otimes \mathbf{m}_{0}^{\alpha}
$$

(see e.g. James and Hane, 2000).

\subsection{Thermodynamic Considerations}

The framework of the thermodynamics with internal variables, as used by Rice (1971), to describe inelastic behavior resulting from plastic behavior of metals, is applied to describe the inelastic behavior of shape-memory alloys resulting from the phase transformation. The internal variables in this thermodynamic framework are the volume fractions of the constituent phases of the RVE. The thermodynamic state variables which describe the state of the system are the Green-Lagrange strain, $\mathbf{E}$, the temperature, $\theta$, and the internal variables, $\xi^{\alpha}$. Using these variables the change in internal energy, $u$, can be written as

$$
\dot{u}=\mathbf{S} \cdot \dot{\mathbf{E}}-\sum_{\alpha=1}^{N} f^{\alpha} \dot{\xi}^{\alpha}+\theta \dot{\eta},
$$

where $\eta$ is the entropy per unit volume, $\mathbf{S} \cdot \dot{\mathbf{E}}$ is the work increment per unit volume, and $f^{\alpha}$ is the force thermodynamically conjugate to the microstructural rearrangement. The Helmholtz free energy is written as

$$
\psi=u-\theta \eta
$$

where $\psi=\psi(\mathbf{E}, \theta, \xi)$. The stress, $f^{\alpha}$, and entropy are defined in terms of the free energy as

$$
\mathbf{S}=\frac{\partial \psi}{\partial \mathbf{E}}, f^{\alpha}=-\frac{\partial \psi}{\partial \xi^{\alpha}}, \eta=-\frac{\partial \psi}{\partial \theta}
$$

It is useful to express the Helmholtz energy as a function of the elastic Green-Lagrange strain, where the elastic strain is defined as

$$
\tilde{\mathbf{E}}^{\mathrm{e}}=1 / 2\left(\mathbf{F}^{\mathrm{e}^{\mathrm{T}}} \mathbf{F}^{\mathrm{e}}-\mathbf{I}\right) .
$$


The approximation to the free energy using the expression for the elastic strain is written as

$$
\hat{\psi}\left(\tilde{\mathbf{E}}^{\mathrm{e}}, \theta, \xi\right)=\frac{1}{2}\left|\mathbf{F}^{\mathrm{tr}}\right| \tilde{\mathbf{S}} \cdot \tilde{\mathbf{E}}^{\mathrm{e}}-c \theta \log \frac{\theta}{\theta_{\mathrm{T}}}-\sum_{\alpha=1}^{N} \xi^{\alpha} \frac{\lambda_{\mathrm{T}}}{\theta_{\mathrm{T}}}\left(\theta-\theta_{\mathrm{T}}\right),
$$

where $\tilde{\mathbf{S}}$ is the second Piola-Kirchhoff stress in the intermediate configuration, which can be written as

$$
\tilde{\mathbf{S}}=\left|\mathbf{F}^{\operatorname{tr}}\right| \frac{\partial \hat{\psi}\left(\tilde{\mathbf{E}}^{\mathrm{e}}, \theta, \xi\right)}{\partial \tilde{\mathbf{E}}^{\mathrm{e}}} .
$$

It is assumed that if the elastic deformation from the intermediate configuration to the current configuration is "small", then $\tilde{\mathbf{S}}$ can be written as ${ }^{1}$

$$
\tilde{\mathbf{S}}=\tilde{\mathbf{C}}^{\mathrm{eff}}(\xi) \cdot \tilde{\mathbf{E}}^{\mathrm{e}} \text {. }
$$

Following Rice's (1971) framework and introducing the finite strain kinematics in (2), the multi-phase composite kinematics in (6), and the definition of the free energy in (12), the form for the force that is thermodynamically conjugate to the phase transformation (which is also taken to be the driving force for the phase transformation) is written as ${ }^{2}$

$$
f^{\alpha}=\left|\mathbf{F}^{\mathrm{tr}}\right| \mathbf{F}^{\mathrm{e}^{\mathrm{T}}} \mathbf{F}^{\mathrm{e}} \tilde{\mathbf{S}} \cdot\left(\boldsymbol{\gamma}_{\mathbf{0}}^{\alpha} \mathbf{F}^{\mathrm{tr}^{-1}}\right)-\frac{1}{2} \frac{\partial}{\partial \xi^{\alpha}}\left(\left|\mathbf{F}^{\mathrm{tr}}\right| \tilde{\mathbf{E}}^{\mathrm{e}} \cdot \tilde{\mathbf{C}}^{\mathrm{eff}} \cdot \tilde{\mathbf{E}}^{\mathrm{e}}\right)-\frac{\lambda_{\mathrm{T}}}{\theta_{\mathrm{T}}}\left(\theta-\theta_{\mathrm{T}}\right) .
$$

\subsection{Inelastic Constitutive Relations for Kinetics}

Kinetics refers to the description of the evolution of the phase transformation. The notion of a driving force can be used to describe the kinetics of the phase transformation. A simple approach in modeling the nucleation is to assume that there is a known critical value of the driving force, $f_{\mathrm{cr}}^{\alpha}$, such that the forward transformation occurs when $f^{\alpha} \geq f_{\mathrm{cr}}^{\alpha}$, and the reverse transformation occurs when $f^{\alpha} \leq-f_{\text {cr }}^{\alpha}$.

\footnotetext{
${ }^{1}$ Note that the product $\mathbf{A B}=A_{i k} B_{k j}$ and the product $\mathbf{A} \cdot \mathbf{B}=A_{i j} B_{i j} \cdot$

${ }^{2}$ Equation (15) written in index notation is $f^{\alpha}=\left|\mathbf{F}^{\mathrm{tr}}\right| F_{j i}^{\mathrm{e}} F_{j k}^{\mathrm{e}} \tilde{S}_{k l} \gamma_{0, i m}^{\alpha} F_{m l}^{\mathrm{tr}^{-1}}-\frac{1}{2} \frac{\partial}{\partial \xi^{\alpha}}\left(\left|\mathbf{F}^{\mathrm{tr}}\right| \tilde{E}_{i j}^{\mathrm{e}} \tilde{\mathrm{C}}_{i j k l}^{\mathrm{eff}} \tilde{E}_{k l}^{\mathrm{e}}\right)-\frac{\lambda_{\mathrm{T}}}{\theta_{\mathrm{T}}}\left(\theta-\theta_{\mathrm{T}}\right)$
} 
The approach taken to model the evolution of the internal variables using a ratedependent approach such that the rate at which the transformation occurs is fully specified from the driving force. The equation that describes the transformation kinetics is

$$
\dot{\xi}^{\alpha}=\operatorname{sign}\left(f^{\alpha}\right) \dot{\xi}_{0}^{\alpha}\left(\mid \frac{f^{\alpha}}{f_{\mathrm{cr}}^{\alpha}}\right)^{m}
$$

where $\xi_{0}^{\alpha}$ is a constant and $m$ controls the rate sensitivity.

\section{Numerical Modeling}

In order for the UMAT to be used for large-scale Taylor (Taylor, 1938) type polycrystal simulations, an implicit time integration scheme is necessary so that relatively large time steps can be taken. Previously, an explicit integration scheme was used to integrate the constitutive equations within the UMAT. The explicit scheme required very small time steps, and therefore even small-scale problems required many iterations, making it impractical for large-scale simulations. What follows in this section is the description of the backward Newton implicit method used to compute the value of the changes in the volume fractions, $\Delta \xi^{\alpha}$. See Appendix II for the source code that implements this integration scheme.

Before the integration scheme can be discussed, it is necessary to describe how the ABAQUS/Standard UMAT works. The input to the UMAT at time $\tau$ is the deformation gradient at time $\tau, \mathbf{F}(\tau)$, the deformation gradient at time $t, \mathbf{F}(t)$, and the stored set of solution dependent state variables, at time $t: \xi^{\alpha}(t)$ and $\mathbf{F}^{\mathrm{tr}}(t)$ (where $\tau=t+\Delta t$ ). The required output from the UMAT is the Cauchy stress, $\mathbf{T}(\tau)$, and the updated state variables, $\xi^{\alpha}(\tau)$ and $\mathbf{F}^{\mathrm{tr}}(\tau)$.

The implicit algorithm is as follows: enter the UMAT with $\mathbf{F}(\tau), \mathbf{F}(t), \mathbf{F}^{\mathrm{tr}}(t)$, and $\xi^{\alpha}(t)$, and compute the elastic trial values of $\mathbf{F}^{\text {tr }}(\tau)$ and $\tilde{\mathbf{S}}(\tau)$. Then compute the potentially forward active transformation systems as those that satisfy

$$
f^{\alpha} \geq f_{\text {cr }}^{\alpha} \quad 0 \leq \xi^{\alpha}<1 \quad 0 \leq \xi<1,
$$

and the potentially reverse active transformation system as those that satisfy

$$
f^{\alpha} \leq-f_{\text {cr }}^{\alpha} \quad 0<\xi^{\alpha} \leq 1 \quad 0<\xi \leq 1 .
$$


If the number of potentially active systems is greater than zero, then compute the driving force, $f^{\alpha}$ given in (15) and the driving force based on the inverse of the kinetic equation, $f_{K}^{\alpha}$, where

$$
f_{K}^{\alpha}=\operatorname{sign}\left(\dot{\xi}^{\alpha}\right) f_{\mathrm{cr}}^{\alpha}\left(\left|\frac{\dot{\xi}^{\alpha}}{\dot{\xi}_{0}^{\alpha}}\right|\right)^{1 / m} .
$$

The purpose of the implicit integration algorithm is to compute the correction the volume fraction change, $\Delta \xi_{\mathrm{c}}^{\alpha}$, such that $f^{\alpha}=f_{K}^{\alpha}$. This condition is ensured by computing the correction to the volume fraction change by solving the equation

$$
\sum_{\beta=1}^{N}\left(\frac{\partial f^{\alpha}}{\partial \Delta \xi^{\beta}}-\delta^{\alpha \beta} \frac{\partial f_{K}^{\alpha}}{\partial \Delta \xi^{\beta}}\right) \Delta \xi_{\mathrm{c}}^{\alpha}-\left(f^{\alpha}-f_{K}^{\alpha}\right)=0 .
$$

The updated volume fractions are then computed as

$$
\begin{gathered}
\Delta \xi_{i}^{\alpha}(\tau)=\Delta \xi_{i-1}^{\alpha}(\tau)+\Delta \xi_{\mathrm{c}}^{\alpha}(\tau) \\
\xi^{\alpha}(\tau)=\xi^{\alpha}(t)+\Delta \xi_{i}^{\alpha}(\tau) \\
\dot{\xi}^{\alpha}(\tau)=\Delta \xi_{i}^{\alpha}(\tau) / \Delta t
\end{gathered}
$$

where $i$ refers to the Newton iteration number. The Newton iteration continues until both of the following two constraints are satisfied

$$
\begin{gathered}
\left|\Delta \xi_{\mathrm{c}}^{\alpha}\right| \leq \mathrm{err} \\
\left|f^{\alpha}-f_{K}^{\alpha}\right| \leq \mathrm{err}
\end{gathered}
$$

After the Newton iteration has converged, the latest values of the volume fractions are used to update the values of $\mathbf{F}^{\mathrm{tr}}(\tau)$ and the Cauchy stress, $\mathbf{T}(\tau)$, and the analysis proceeds to the next time step. The implicit integration algorithm is outlined in the flow chart in Figure 1.

Implementing the Newton implicit time integration scheme reduces the number of time steps required to complete a simulation drastically. For example, during the simulation ${ }^{3}$ of a single crystal under a tensile (and compressive) loading-unloading cycle up to $7 \%$ strain (see Figure 2 for simulation results), the implicit scheme reduces the number of necessary time steps by a factor of more than 100 .

\footnotetext{
${ }^{3}$ In this simulation: $\xi_{0}^{\alpha}=10^{-3}, f_{c r}^{\alpha}=10 \mathrm{MJ} / \mathrm{m}^{3}$, and $m=100$.
} 


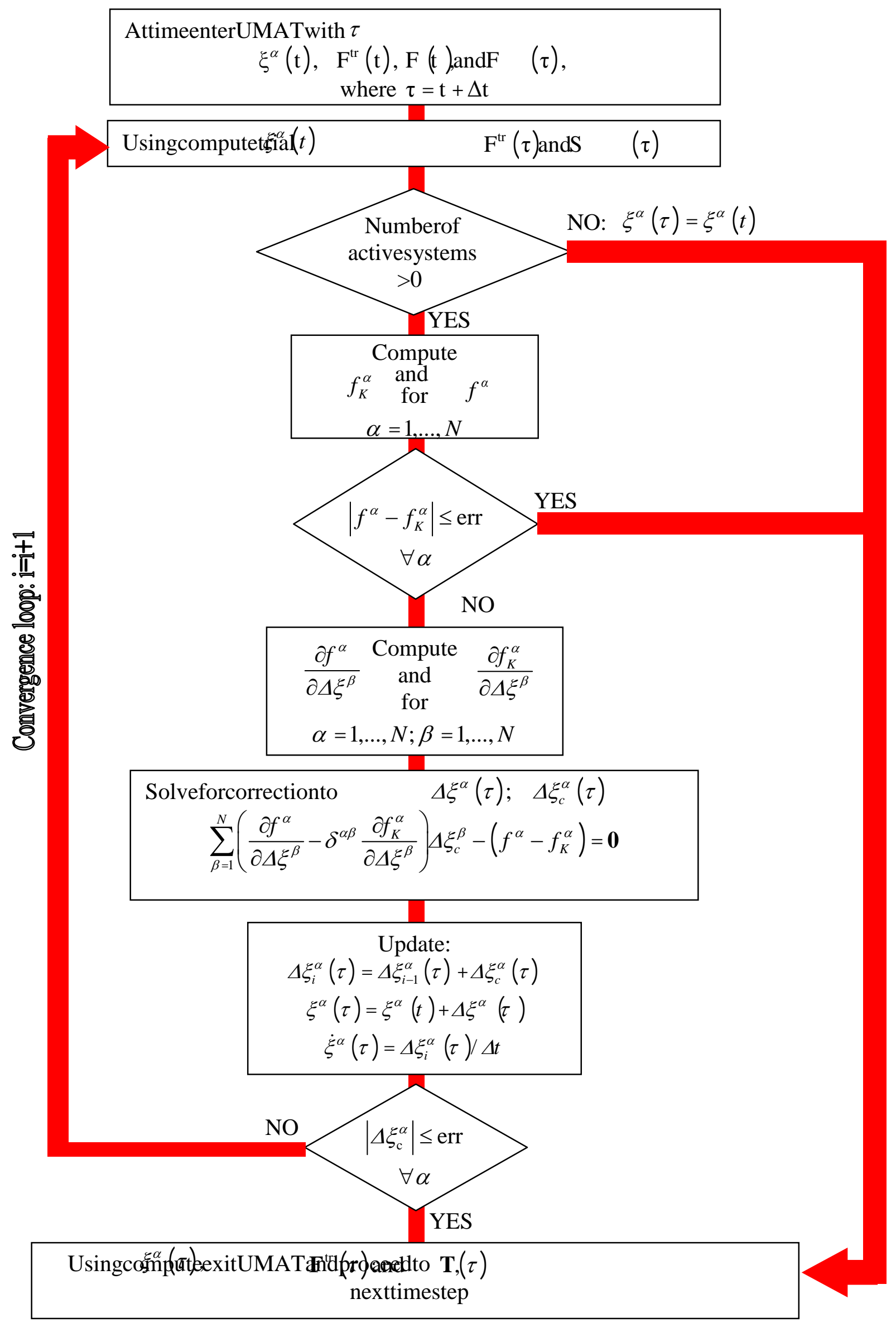

Figure 1: Flow chart of the implicit time integration scheme used in the ABAQUS/UMAT. 


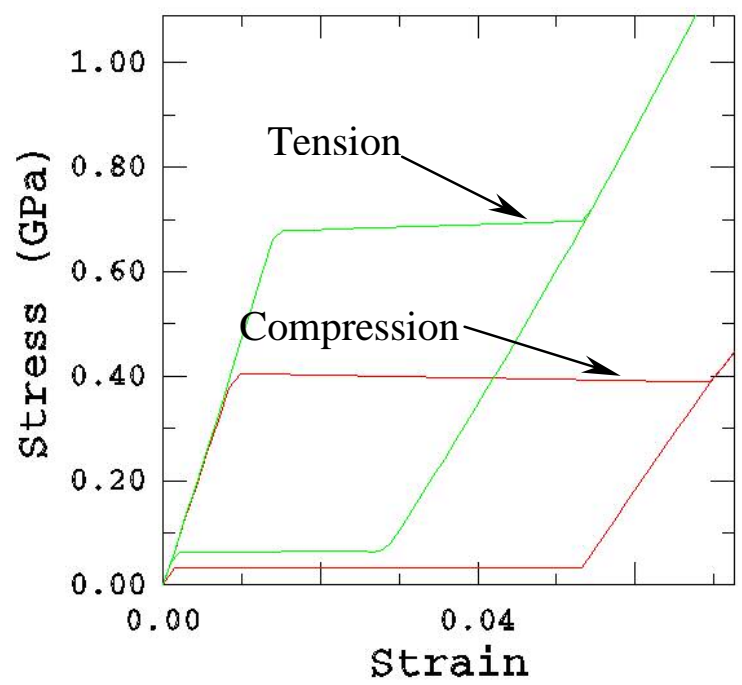

Figure 2: Pseudoelastic response of a single crystal ([100] orientation) under uniaxial tension (green curve) and uniaxial compression (red curve) at $285 \mathrm{~K}$.

These simulations were performed with implicit time integration and completed in approximately 70 times steps. This is a great improvement over the explicit scheme, which took approximately 10,000 time steps to perform the same calculation.

\section{Future Work}

Now that the implicit time integration scheme has been implemented, large-scale Taylor type (Taylor, 1938) polycrystal simulations can be performed. Plans for future work include using the implicit version of the UMAT to perform simulations on a thin walled NiTi tube. Tension, torsion, as well as combined tension-torsion (both proportional and non-proportional) loadings will be considered and compared to experimental results. The finite-element mesh that will be used to simulate the thin-walled tube is shown in Figure 3(a), the texture to be used is shown in Figure 3(b). See Appendix II for the input file for the thin-walled tube.

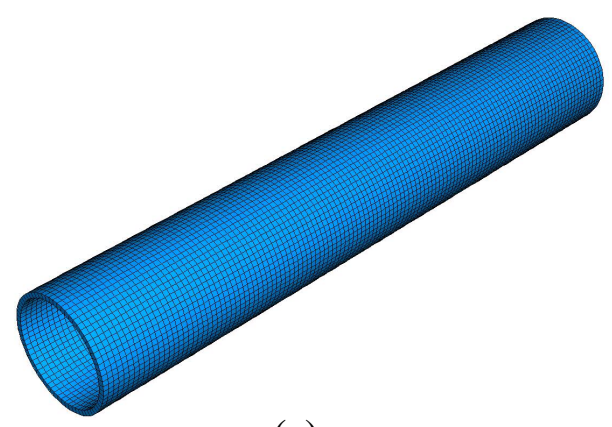

(a)

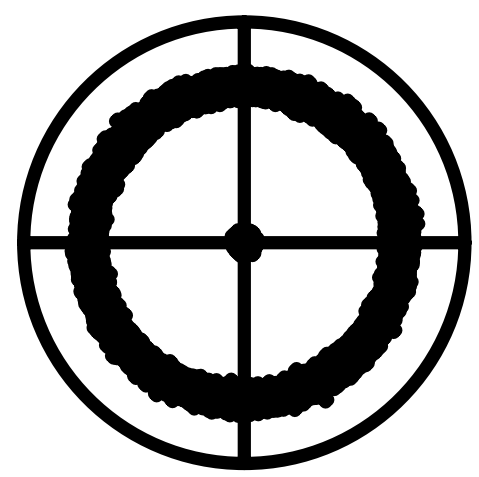

(b)

Figure 3: The finite-element mesh to be used in the future simulation of a textured thin-walled NiTi tube is shown in (a). The mesh contains 8,000 elements and is $25 \mathrm{~mm}$ long. The pole figure showing the strong [111] fiber texture that will be used in the tube simulation is shown in (b). 


\section{References}

[1] ABAQUS (2003). Reference Manuals, Providence, RI.

[2] James, R.D. and Hane, K.F. (2000). Martensitic transformations and shape-memory materials. Acta mater., 48, pp. 197-222.

[3] Jannetti, C., Bassani, J.L., Turteltaub, S. (2003). Three-dimensional finite-element simulation of shape-memory alloys using a thermodynamically-based theory of martensitic phase transformations. Seventh United States National Congress on Computational Mechanics, Albuquerque, NM.

[4] Reuss, A. (1929). Calculation of the flow limits of mixed crystals on the basis of the plasticity of monocrystals. Z. angnew. Math. Mech. 19, pp. 49-58.

[5] Rice, J.R. (1971). Inelastic constitutive relations for solids: an internal variable theory and its application to metal plasticity. J. Mech. Phys. Solids, 19, pp. 433-455.

[6] Rice, J.R. (1975). Continuum mechanics and thermodynamics of plasticity in relation to microscale deformation mechanisms, in Constitutive equations in plasticity (A.S. Argon, editor), pp. 23-79, MIT press, Cambridge, MA.

[7] Taylor, G. (1938). Plastic strain in metals. J. Inst. Met, 62, pp. 30-324. 